\title{
Research on Hybrid Online Course Supervision Platform Based on Data Analysis
}

\author{
Gang Chen", Zhicheng Li, Jiacheng Zhu
}

\begin{abstract}
School of Mathematics and Computer Science, Jianghan University, Wuhan, China, 430056
*Corresponding author
\end{abstract}

\begin{abstract}
In this paper, an example of the supervision platform in mixed teaching is used to study the key issues of online and offline mixing mechanisms. Explore the construction of a hybrid teaching data center through a computer supervision platform, use data analysis methods and machine learning algorithms to construct evaluation indicators and evaluation models for student learning status, and implement personalized teaching for students. This article also introduces in detail the supervision process and supervision methods of Jianghan University's "University Computer Fundamentals" course to realize the hierarchical teaching of students, and proposes a mixed teaching mode under the environment of large engineering classes.
\end{abstract}

Keywords: Mixed teaching, Supervision platform, Supervision methods

\section{基于数据分析的混合在线课程督学平台研究}

陈刚"，李支成，朱家成

江汉大学数学与计算机科学学院, 武汉, 中国, 430056

“通讯作者

\section{中文摘要}

本文通过一个混合教学中督学平台实例, 研究线上线下混合机制关键问题。探讨通过计算机督学平台 构建混合教学数据中心, 采用数据分析手段和机器学习算法, 构建学生学习状态评价指标和评价模型, 实现学生的个性化教学。本文还详细介绍了江汉大学 “大学计算机基础” 课程实现学生分层教学的督 学流程和督学手段, 提出了一种工科大班环境下的混合教学模式。

混合教学是线上线下结合的教学模式, 线上教学通常采用MOOC或SPOOC教学, 教师把预先录制好的教学 视频、线上作业、线上测试等放到互联网平台, 实现学生自主学习; 线下课堂教学同步保留, 但线下 课堂目标变成督学, 即教师不是以讲课为主要工作, 而是需要通过获取学生各种学习数据, 进行有效 分析, 个性化指导学生学习。

这种混合教学模式能解决传统教学中普遍教学学时不够、教师无法关注学生个性化需求、教学同质化 等问题。学生是学习主体, 自主学习是主要特征; 教师是教学主体, 教师关注课堂教学质量, 关注课 程目标达成度，关注学生个性化需求。双主体可以保证取得较好的教学效果。

这种教学模式的主要难点问题有两个, 一是线上线下课堂的融合机制, 判断学生的学习状态是重点问 题; 二是如何针对不同学习状态的学生进行督学并提供不同的学习路径。

江汉大学从2017年开始按照这种模式对 “大学计算机基础” 课程开展教学，取得较好效果。从每年的 4000学生采用同一题库同一考核方式最后结果来看, 未开展混合教学的2016年, 该课程的不及格学生 有 590 人； 2017 年拿出了 1300 人进行混合试点, 补考学生为 179 人； 2018 年全部采用混合教学，补考人 数为 26 人。 
在课程实践中, 通过设计的 “线上学习平台” + “线下督学平台” 的双平台模式, 较好解决混合教学的 关键问题。线上学习平台是在超星平台构建的学习资源, 包括课程视频、测验、作业、讨论、扩展学 习资料等。督学平台是教师教学的主要平台, 由于学生学习数据已经全部数字化, 数据量巨大, 再加 上学生人数较多的因素, 开发相应的计算机系统来处理大量数据, 同时进行实时数据分析, 是混合教 学中必然的选择。

关键词：混合教学；督学平台；督学手段

\section{1. 督学平台功能分析}

督学平台是混合教学的数据集成分析平台, 通过集 成线上平台、课上测试系统数据以及课堂实验、点名、 随堂练习等学习过程数据, 使教师能实时获取学生当前 学习状况数据。督学平台应该还能够提供数据分析功能, 教师能根据分析结果对学生进行分层。

督学平台作为教师课堂教学的主要工具, 主要体系 如图1:

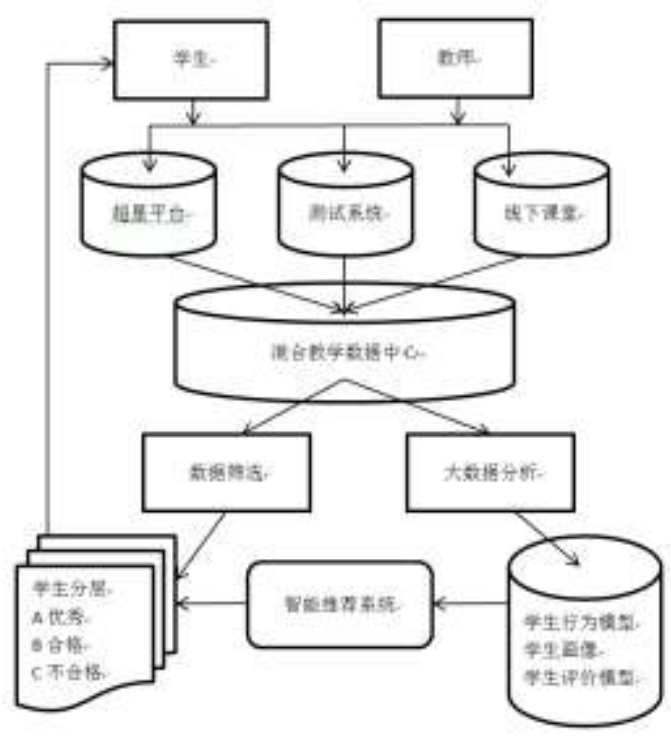

图 1 督学平台体系

在督学体系中, 教师是主体, 通过线下课堂对学生 采用各种教学手段, 集成学生的学习状态数据, 进行数 据分析, 达到学生区分教学的目标。督学体系的核心内 容包括:

\section{1. 混合教学数据中心}

数据中心是混合教学的核心, 目标是集成能收集到 的各项学生学习数据。包括线下课堂督学时学生在线上 自主学习状况分析, 课堂实时测试成绩, 课堂点名、实 验、练习等各方面资源。

大学计算机基础课程线上数据是从超星平台实时 一键导出数据, 如图2。

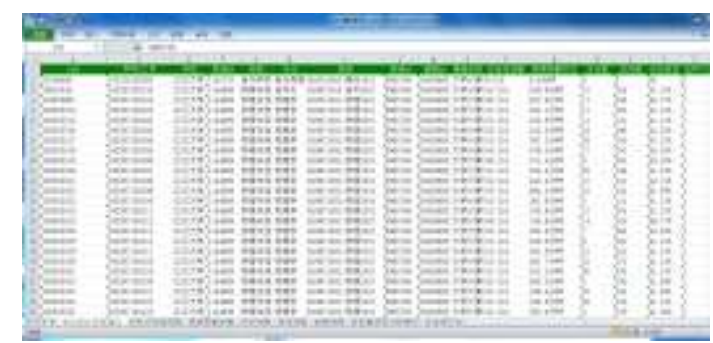

图 2 超星线上平台导出数据

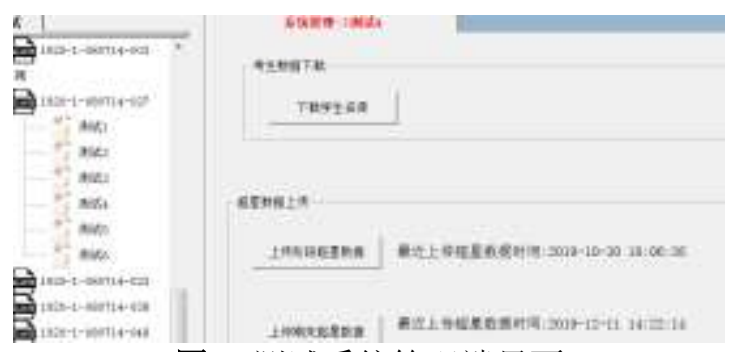

图 3 测试系统管理端界面

测试系统是自主研发的考试系统六个单元测试, 如 图3。线下数据是独立开发的自主学习平台数据, 如图4。

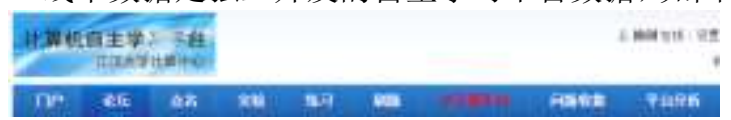

图 4 自主平台功能图

三部分数据通过自主学习平台实现集成。

例如 2019级080714-027班部分学生第四单元从督 学系统导出考评表如图5:

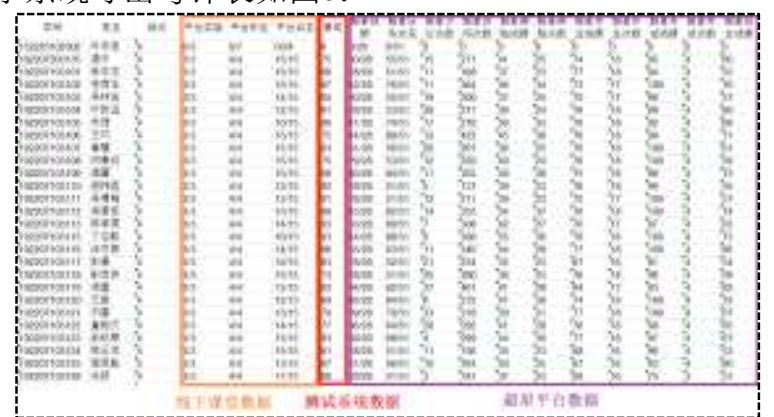

图 5 数据集成样表 


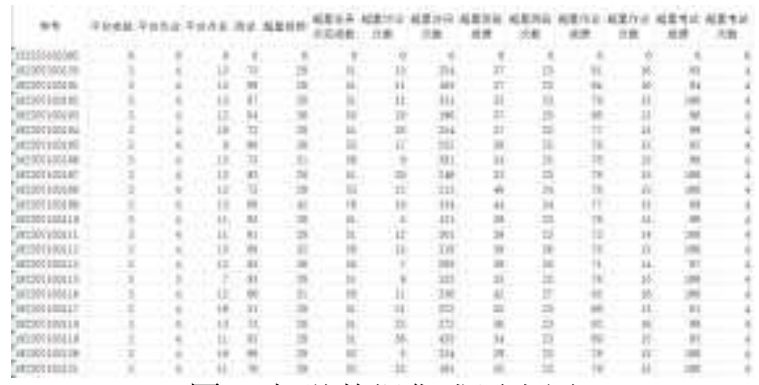

图 6 督学数据集成原表图

\section{2. 数据分析模块}

数据分析有两种途径, 简单模式采用数据笁选模式 对学生分层, 大数据分析模式因为需要有一定的数据积 累, 目前已经启动研究。

大学计算机基础课程的混合教学模式通常采用数 据篎选模式，教师在单元测试点对学生进行第一次考评。 考评指标包括阶段到课、实验、练习分析数据、测试系 统课堂考试原始成绩、超星分析数据, 整合为一张数据 表。例如2019级080714-027班部分学生第四单元从督学 系统导出考评表如图6。

教师可以根据预设的考评指标对学生是否达标进 行筛选, 重点是䇻选出未达标的学生, 突出显示其未达 标指标。单元考评结果样表如图7:

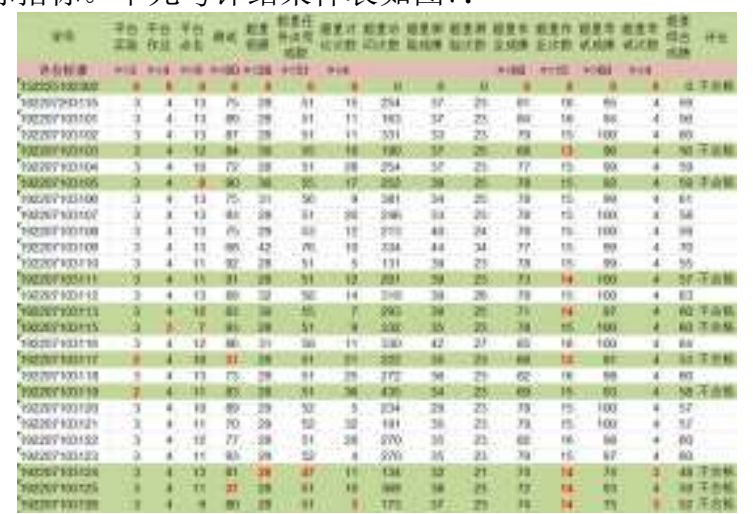

图 7 首次督学分层图

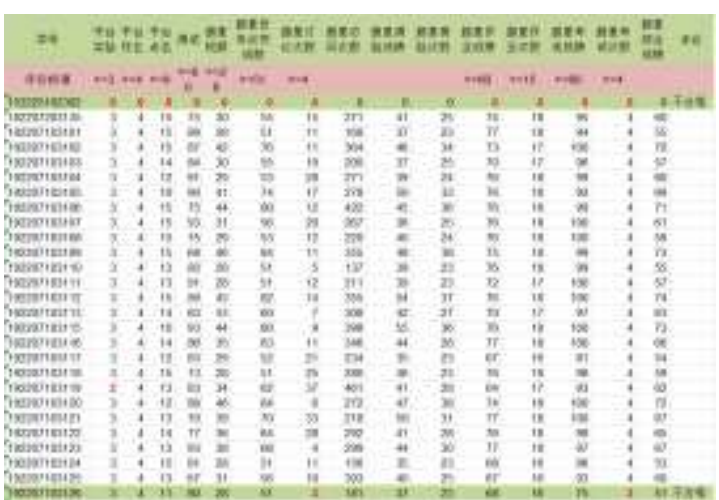

图 8 阶段督学结束结果对比
经过教师每周第二次课督学后, 教师对学生数据进 行阶段结束最终数据考评, 确定阶段单元督学效果。本 单元最终数据样表如图8。

\section{3. 督学流程}

督学结果由教师选择公布在超星平台通知区、讨论 区或者教师 $\mathrm{QQ}$ 群。要求教师有相应的成绩记载期末统一 收集。例如发布在超星平台讨论区的单元督学数据如图 9:

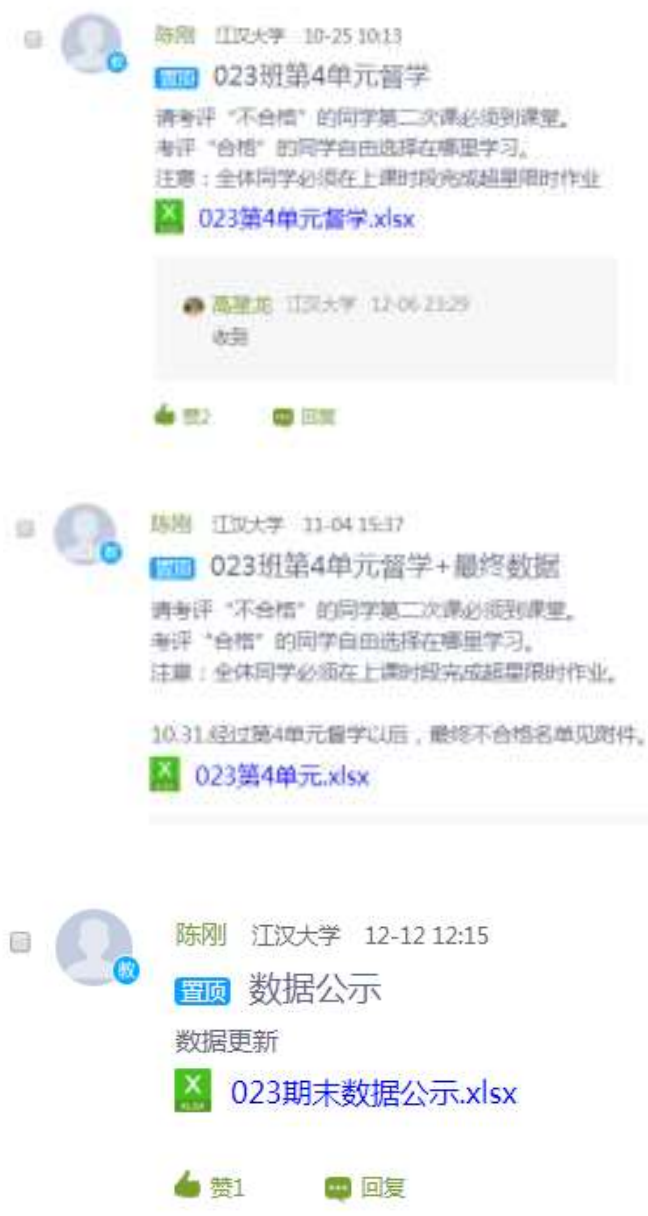

图 9 督学通知发布示例图

教师可以根据教学需要对能够达标的学生分多层 教学, 一般分优秀和合格, 分别提供不同难度的教学超 星学习内容供学生学习。

例如: Exce1数据管理这一章节, 内容分三档。

一是每个学生必须完成的实验指导书规定的实验 六, 放在线下自主平台, 是对应期末考试的最低要求。 二是超星限时作业中教师布置的超星限时专项训练, 供 中等学生的选择完成。三是超星作业中的Exce1综合练 习, 对应难度为全国计算机等级考试二级水平, 供优秀 学生选做。三挡内容如图10。 


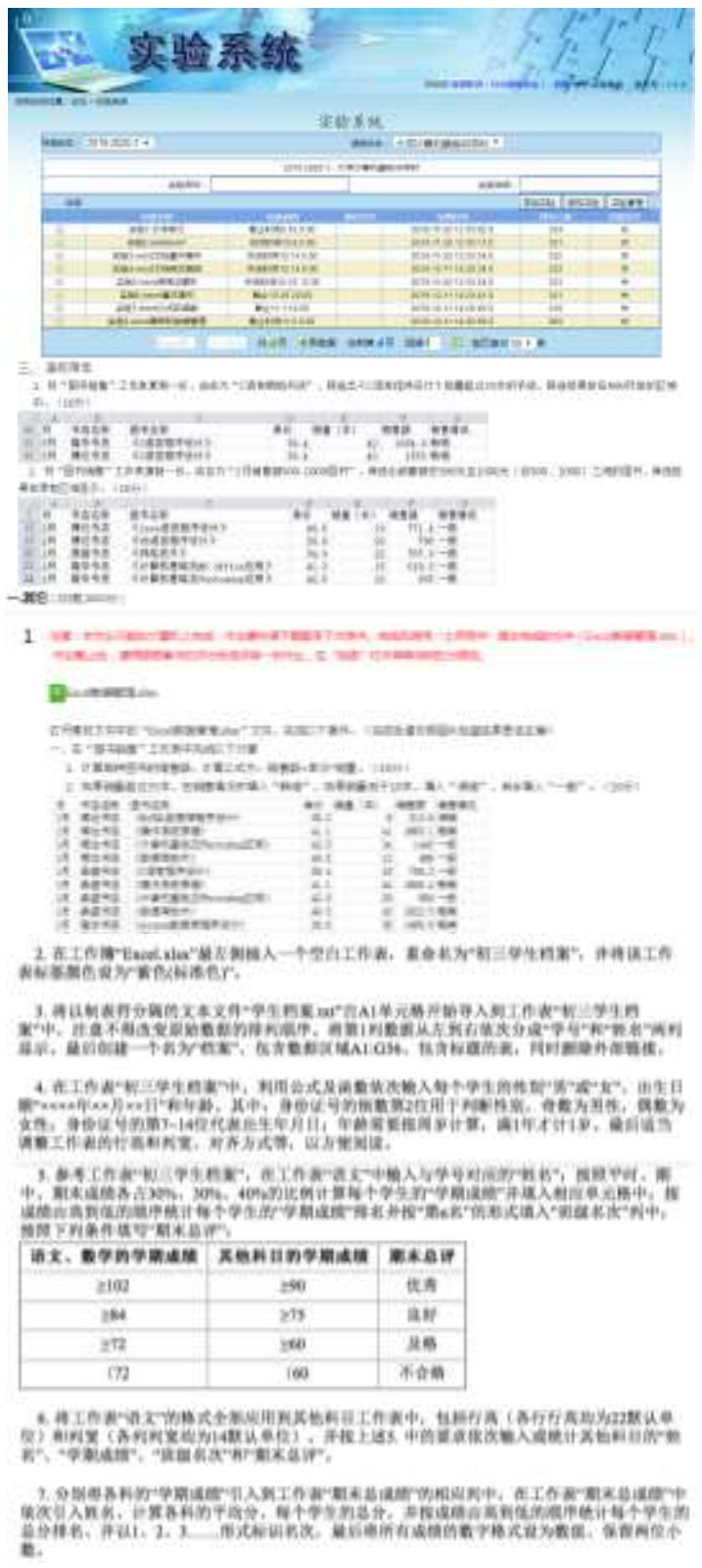

图 10 低中高三级级要求的专项训练示例

线上课通过超星限时作业、PBL项目等手段进行检 测和评价。如图11。

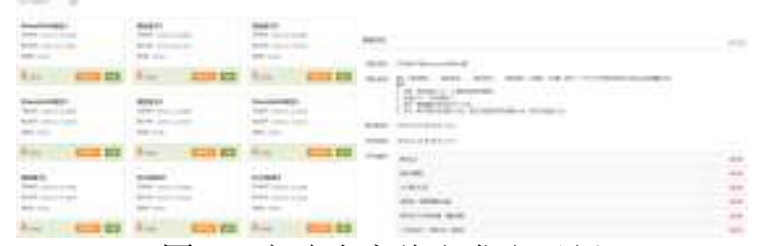

图 11 高阶内容线上发布示例

\section{2. 督学平台问题探讨}

在督学平台开发中, 存在如下关键问题。

\section{1. 基于 $B S$ 的全自动考试系统}

在混合教学中关键问题是获取学生学生状态, 一般 教师采用课堂提问、讨论、抢答等手段只能获得部分学 生的状态, 计算机测试系统能实时客观真实的反映学生 的学习状态, 是混合教学的核心工具。

混合教学的测试系统具有实时性和客观特点, 需要 解决 2 个问题, 一是主观题的自动评分问题。课程性质 不同评分模式不同, 对于大学计算机基础课程而言, 必 须解决汉字录入、Windows、Word、Excel、PowerPoint、 网络操作题的自动评分。二是互联网考试问题, 互联网 考试从现有开发技术上有一定的挑战, 重点需要解决身 份识别、BS架构数据传递、并发控制等问题。

目前 “大学计算机基础” 课程已经基本实现了各 种操作题的评分问题, 基于BS架构测试系统正在开发。

\section{2. 基于机器学习的数据分析模型}

混合教学数据中心经过累积数据, 可以用于对学生 学习状态进行分析。目前的数据篮选模式只能解决表面 问题, 需要研究采用大数据分析方法, 不断构建和完善 学生学习数据, 构建学生学习行为分析模型, 实现学生 画像功能, 为实现智能推荐提供依据。

采用机器学习算法, 形成分阶段的学生学习评价模 型, 为教师个性化教学提供直接依据。

目前 “大学计算机基础” 课程已经有老师开始研究 各个阶段学生评价的核心指标体系, 需要在教学中接受 检验。

\section{3. 学生学习路径的智能规划方案}

在数据分析的基础上, 可以通过丰富课程体系, 完 善课程内容, 使各种高阶评价体系形成规范, 这时可以 通过开发智能推荐系统, 为学生提供个性化的学习方案。

目前, “大学计算机基础” 课程计划开发虚拟仿真 实验系统, 打造课程的综合实验体系, 根据学生的学习 兴趣、学习习惯, 构建一个层次丰富、客观方便使用的 互联网仿真系统。

\section{3. 结语}

在混合教学中, 教学过程与计算机技术深度结合, 教学逐步实现数字化, 教学资源的数据化使教学流程中 数据分析成为重要教学手段。督学平台通过构建混合教 学数据中心, 可以辅助教师获取学生的实时学习状态, 并通过系统提供的各种工具, 将教学资源与教学过程连 接成为一个智能紧密有机的整体, 是混合教学中具有不 可替代的重要作用。 


\section{致谢}

本文为湖北省级教学研究项目 “基于数据分析的 分层混合教学模式研究”（编号2016277），2017湖北 省教育科学规划课题 “SPOC混合教学下学生学习行为 分析和预测研究” (2017GB030), 武汉市教育科学 “十 三五” 规划2018重点课题 “基于SPOC的混合教学交互 过程研究”（2018A047）的阶段性成果之一。

\section{REFERENCES}

[1] Gang CHEN. Case Study of Hierarchical Mixed Teaching Model Based on Data Analysis. 2018 International Conference on Education Reform and Management Science (ERMS2018). 2018.5. p82-86.

[2] Gang CHEN. Research on Teaching Effectiveness Index System in Mixed Teaching Mode. 2018 3rd International Conference on Education, Management and Systems Engineering (EMSE2018). 2018.11. p343-348.

[3] Gang CHEN. Research on the Inspector System under the Mixed Teaching Mode of Online Courses. 2019 International Conference on Management Innovation, Education Reform and Applied Social Science (MIERASS2019).2019.4. p223-227. 